\title{
Plasma-wall interaction and plasma behaviour in the non-boronised all tungsten ASDEX Upgrade
}

\author{
R. Dux, V. Bobkov, A. Herrmann, A. Janzer, A. Kallenbach, R. Neu, \\ M. Mayer, H.W. Müller, R. Pugno, T. Pütterich, V. Rohde,
} A.C.C. Sips, ASDEX Upgrade Team

Max-Planck-Institut für Plasmaphysik, EURATOM Ass., D-85748 Garching, Germany

\begin{abstract}
ASDEX Upgrade has successfully started the second experimental campaign with a full tungsten coverage of the plasma facing components and without using a boronisation for machine conditioning. The tungsten erosion at all relevant positions in the main chamber and the divertor was investigated. The outer divertor is by far the strongest source region, especially in discharges with high divertor temperature in-between ELMs. In the main chamber, the central column is usually the first limiting structure and produces then larger $\mathrm{W}$ erosion fluxes than the outboard limiters. Nevertheless, the tungsten influx from the outboard limiters has a much stronger effect on the tungsten content in the confined plasma. An increase of the available power from the fly-wheel generator allowed for improved $\mathrm{H}$-mode operation at $1 \mathrm{MA}$, and $\mathrm{H}$ factors in the range of 1.2 could be achieved at acceptable $\mathrm{W}$ concentrations of about $2 \times 10^{-5}$.
\end{abstract}

Key words: First wall, Tungsten, Erosion, ELM, Cross field transport, ASDEX Upgrade PACS: 52.40.Hf, 52.25.Vy, 52.55.Rk, 52.55.Fa

Corresponding author: Ralp Dux, Boltzmannstr.2, D-85748 Garching, Germany.

E-mail address: Ralph.Dux@ipp.mpg.de

Tel.+49-89-3299-1256;

fax:+49-89-3299-1812 


\section{Introduction}

In the year 2007, ASDEX Upgrade had the first experimental campaign with full tungsten coverage of all plasma facing components and without any boronisation before or during the campaign [1]. The avoidance of a boronisation created a clean testbed for the post campaign investigation of hydrogen retention, which has drastically reduced and is discussed in detail in Ref.[2]. The newly installed divertor strike point tiles, which had a $200 \mu$ m thick coating [1], behaved very well and showed no failure. Prior to the present 2008 campaign, a wet cleaning of the inner column and the limiters was performed, which caused a further reduction of spectroscopic boron signals below the detection limit. The present campaign was again restarted without a boronisation applying the same conditioning procedures as in 2007 [1], i.e a long (203 h) bakeout of the vessel at $150^{\circ} \mathrm{C}$ and several overnight He glow discharges. Further conditioning was done with plasma discharges and inter-shot He glow discharges. In order to diminish the helium storage in the tungsten [3], the glow discharges in He were finally replaced by glows in deuterium, which quickly reduces the He concentration of the plasma from the $30 \%$ level by a factor of 10 within 5-10 discharges. The technical constraints due to the loss of one fly-wheel generator have been somewhat relaxed by increasing the maximum generator current and the reactive power compensation. Thus, the majority of the discharges could now be run at a plasma current of $1 \mathrm{MA}$ and had up to $14 \mathrm{MW}$ of external heating power.

The following sections concentrate on tungsten erosion in the divertor, which was the last element, to complete the transition to a full $\mathrm{W}$ device. In section 2, campaign integrated erosion in the outer divertor is described, while section 3 discusses time resolved measurements with an emphasis on the strong increase of the erosion during an edge-localised mode (ELM). The main chamber tungsten sources and their influence on the $\mathrm{W}$ concentration in the confined plasma are the topic of section 4. Here again, the impact of ELMs are an important element, which prevent the accumulation of $\mathrm{W}$ in the edge transport barrier. Thus, the achievement of improved $\mathrm{H}-$ mode discharges with good energy confinement at acceptable $\mathrm{W}$ concentrations is the result of balancing energy versus impurity confinement and again the ELMs are an important ingredient as will be shown in section 5 . 


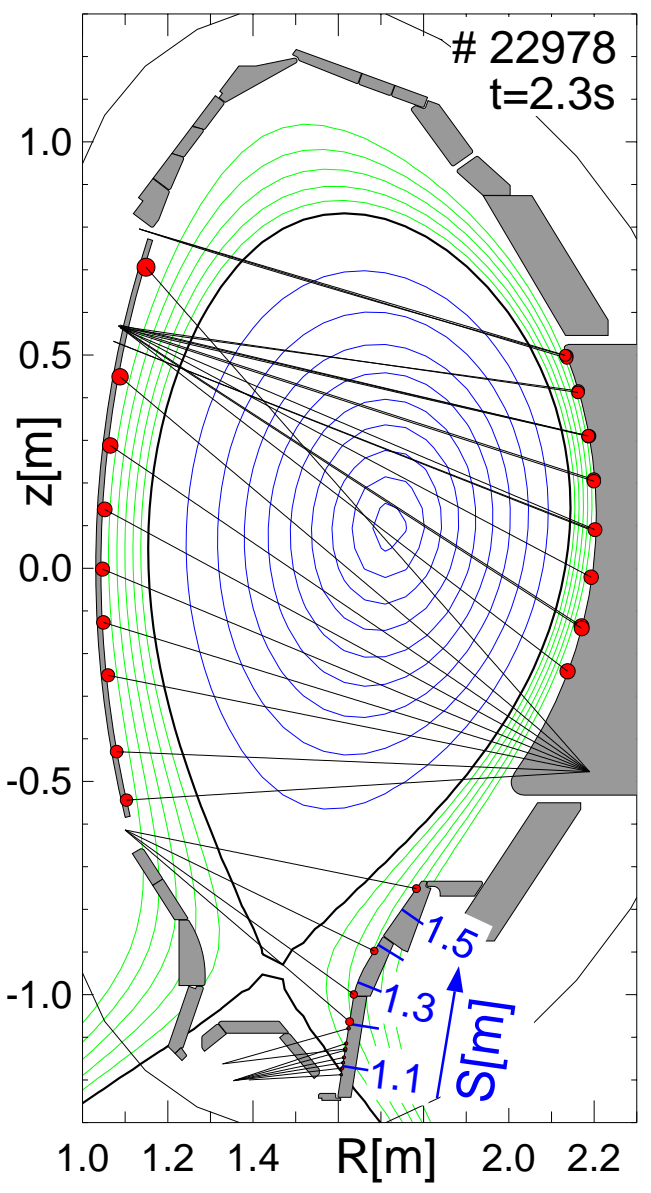

Fig. 1. Cross section of ASDEX Upgrade: The flux surface geometry is shown for \#22978 at 2.3 s including the poloidal projection of all 38 lines-of-sight used for tungsten influx measurements. The red circles indicate the observed spot size at the surface. In the lower outer divertor, the co-ordinate for the poloidal surface contour $S$ is shown.

\section{Campaign integrated tungsten erosion in the outboard divertor}

The largest erosion rates of tungsten due to plasma impact are observed at the strike point tile of the outboard divertor. Two diagnostics are available to quantify the tungsten erosion. A campaign integrated value is delivered by determination of the eroded $\mathrm{W}$ coating thickness using Rutherford-backscattering (RBS) [4]. To this end, marker tiles with a thin tungsten stripe on carbon are employed. The plasma vapor deposited tungsten had a thickness of $d=1.5 \mu \mathrm{m}$ and the layer thickness was measured before and after the 2007 campaign. The difference $\Delta d$ delivers a value for the net erosion. By measuring the deposited tungsten thickness $d_{d e p}$ on the carbon tile away from the $\mathrm{W}$ stripe, an estimate of the gross erosion $\Delta d+d_{d e p}$ is gained, assuming that the same amount of tungsten was also deposited on the stripe. The measurement of 


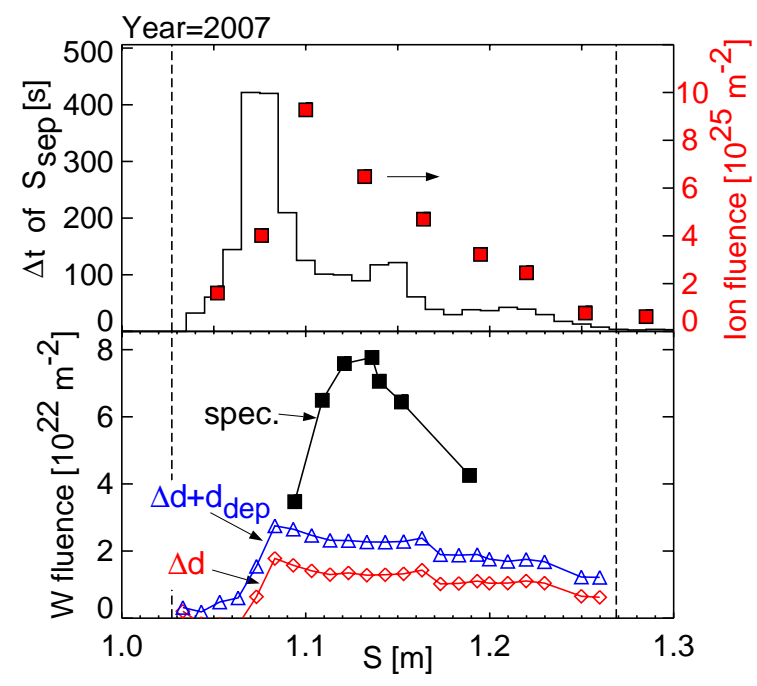

Fig. 2. W erosion at the strike point tile during the 2007 campaign: All profiles are plotted versus the co-ordinate $S$ which follows the poloidal divertor contour. The dashed lines give the tile edges. In the upper graph, the total duration of the outer strike point at a certain tile position and the total impinging ion fluence onto the Langmuir probes is shown. The lower panel compares the spectroscopically determined $\mathrm{W}$ erosion with the values obtained from surface analysis.

$d_{d e p}$ was taken at a toroidal distance of $1 \mathrm{~cm}$ to $\mathrm{W}$ surfaces, where the contribution of promptly redeposited tungsten is small [5]. Time resolved measurements are obtained from spectroscopic measurements. Here, the tungsten influx is monitored by measuring the WI line radiation at $400.9 \mathrm{~nm}$ on a number of lines-of-sight covering the outboard divertor. In the 2007 campaign, seven lines-of-sight were available, and in the 2008 campaign, the influx is routinely measured on 12 lines-of-sight. The observed spots have a diameter of about $2 \mathrm{~cm}$. The measured photon flux density can be transformed into an eroded tungsten flux density using the number of ionisations per emitted photon [6], i. e. the $(S / X B)$ value. For tungsten $(S / X B)=20$ is used [7]. Each eroded $\mathrm{W}$ atom contributes to the spectroscopically determined erosion flux.

For the 2007 campaign, a comparison of the two measurement methods is shown in the lower panel of Fig.2. The thickness change $\Delta d$ from surface analysis is at maximum $280 \mathrm{~nm}$, representing a fluence of $1.8 \times 10^{22} \mathrm{~m}^{-2}$. The total time duration with strike point in the outer divertor was $\Delta t_{t o t}=2317 \mathrm{~s}$ yielding an average erosion rate of $0.12 \mathrm{~nm} \mathrm{~s}^{-1}$. On the microscopic scale, the spatial structure of the erosion is inhomogeneous with predominant erosion on protruding areas. With regard to the spectroscopic data, the campaign integrated W fluence was obtained by integrating the fluxes over the available measurement time $\Delta t_{\text {meas }}=1355 \mathrm{~s}$ and multiplying the 
result with the factor $\Delta t_{\text {tot }} / \Delta t_{\text {meas }}$. The fluences from surface analysis and the spectroscopic fluence are in very good agreement and show the expected relation, i.e. that spectroscopy, which measures all erosion events, yield the highest values. However, the systematic uncertainties of the spectroscopically determined $\mathrm{W}$ fluxes being about a factor of 2 due to absolute calibration and atomic data uncertainties, do not allow to draw conclusions about the prompt redeposition probability, which should be around $90 \%$, since for a typical electron density $n_{e}=10^{20} \mathrm{~m}^{-3}$ the ionisation length of $\mathrm{W}$ is in the range of $0.1-0.2 \mathrm{~mm}$, i.e. much shorter than than the ion gyro radius of about $2 \mathrm{~mm}$ at a magnetic field of $2.5 \mathrm{~T}$. The upper panel of Fig. 2 shows the distribution of the strike point position during the campaign. The integral of the ion saturation current onto the flush mounted Langmuir probes yields a value for the total impinging ion fluence, which is shown for the Langmuir probe positions in the outboard divertor. When dividing the spectroscopic W fluence by the impinging ion fluence, effective $\mathrm{W}$ erosion yields $Y_{\text {eff }}$ of the order of $10^{-3}$ are obtained.

\section{Time resolved tungsten erosion in the outboard divertor}

For time resolved spectroscopic measurements of tungsten erosion, one line-of-sight is routinely recorded with a resolution of $200 \mu \mathrm{s}$, while the remaining 11 channels integrate over $3 \mathrm{~ms}$. The fast tungsten influx measurement shows a strong variation with the bursts of energy and ion flux that are caused by type-I ELMs in the scrape-off layer (SOL) of H-mode plasmas. Examples of the induced influx bursts due to ELMs are given in Fig.3, where the spectroscopic signal rises by a factor of 10 or more.

The time averaged contribution of the ELM induced local flux density shall be defined as $c_{E L M}=\left(\langle\Gamma\rangle-\Gamma_{o f f}\right) /\langle\Gamma\rangle$ where $\langle\Gamma\rangle$ is the temporal average of the influx density over many ELM cycles and $\Gamma_{\text {off }}$ is the offset influx in-between ELMs. $\Gamma_{\text {of } f}$ is depicted with a grey/red line in Fig. 3. The value of $\Gamma_{\text {off }}$ depends on the temperature at the target $T_{e, d i v}$. Time traces of $T_{e, d i v}$ as measured by a triple probe are shown in the lower panels of Fig.3. The Langmuir probe data can not be reliably evaluated during the ELM and the according time spans are left out in the graphs. The offset $\Gamma_{\text {off }}$ is rising with the temperature in-between ELMs. The first column is a case with low recycling and high $T_{e, d i v}$ throughout the ELM cycle, the second example has a 


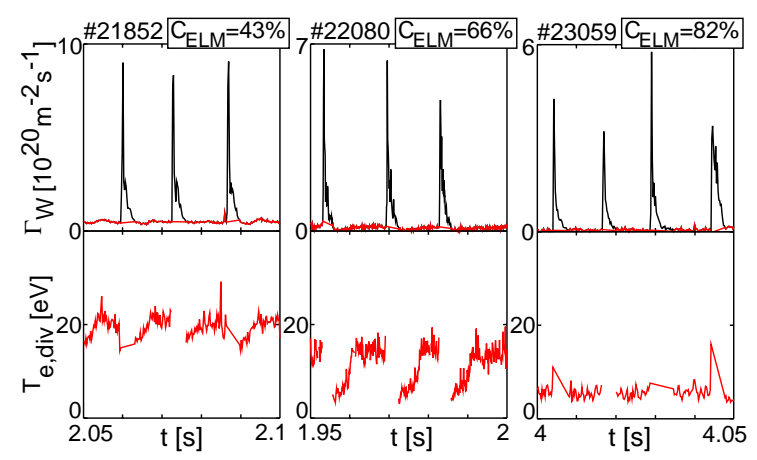

Fig. 3. Time traces of $\mathrm{W}$ influx densities and divertor electron temperatures in the SOL of the outer divertor target during several ELM cycles: Three cases with rising recycling level and consequently lower divertor temperatures in-between ELMs are shown. The red/gray lines highlight the offset signals in-between ELMs. The relative contribution of ELMs to the total time averaged W influx increases with increasing recycling level.

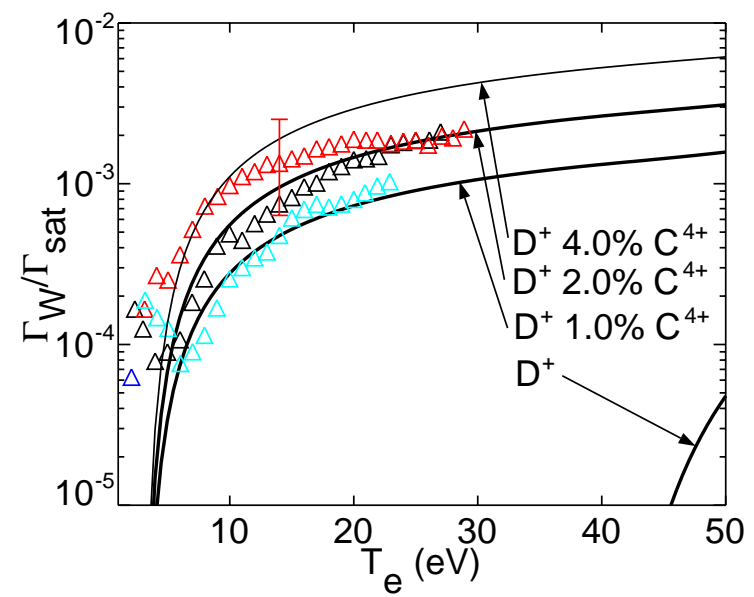

Fig. 4. Effective erosion yield of tungsten for sputtering in-between ELMs.

short high recycling phase with low temperature after the ELM, which lasts during the complete ELM cycle in the last example. The increase of $\Gamma_{o f f}$ with increasing $T_{e, d i v}$ is due to the strong increase of the effective erosion yield up to a temperature of about $T_{e}=12 \mathrm{eV}$ which is stronger than the decrease of the impinging ion flux with rising $T_{e}$. The contribution of ELMs depends mainly on the temperature in-between ELMs and varies between $43 \%$ for the low recycling case to $82 \%$ for the high recycling case.

Fig. 4 shows the measured dependence of the effective erosion yield $Y_{e f f}=\Gamma_{W} / \Gamma_{D}$ on $T_{e}$, where $\Gamma_{D}$ is approximated by the ion saturation flux density $\Gamma_{s a t}$ as measured by Langmuir probes. The data are gained from three H-mode discharges(\#23059,\#21887,\#22920), which document the measured variance of $Y_{\text {eff }}$ for a given temperature. Only the times in-between 


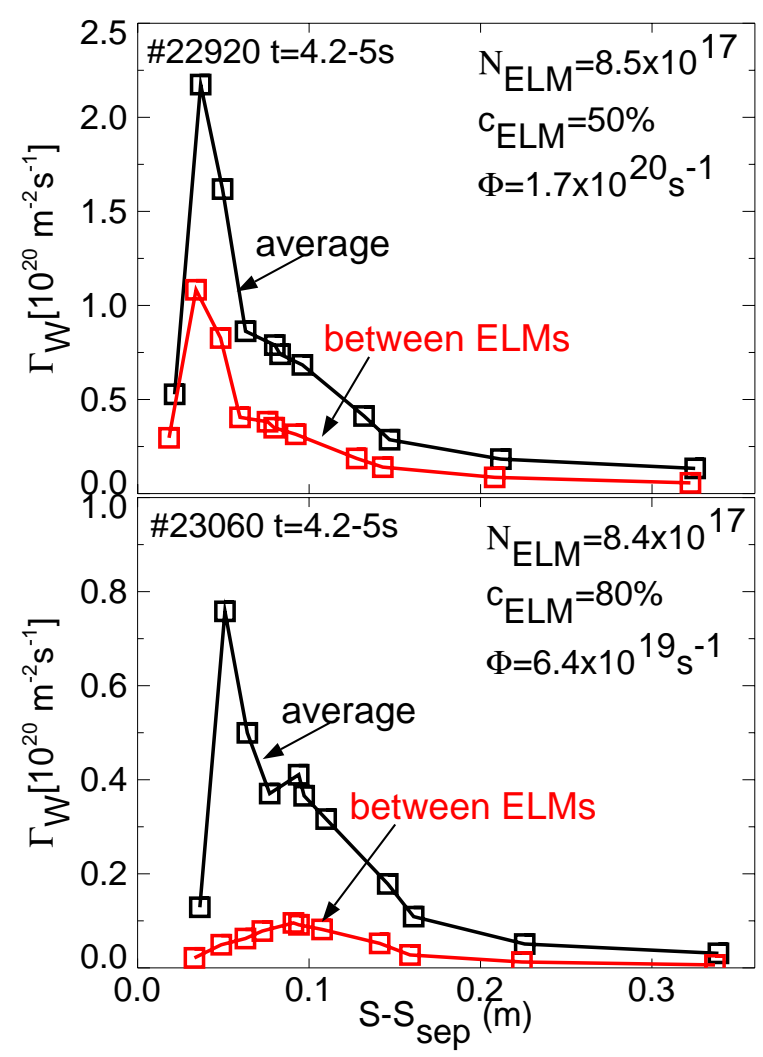

Fig. 5. Profiles of the tungsten influx in the outboard divertor. Between the ELMs, \#22920 has a high divertor temperature of $20 \mathrm{eV}$ near the separatrix, while \#23060 has a low value of $6 \mathrm{eV}$.

ELMs have been taken into account. The measured yields increase sharply with temperature up to about $12 \mathrm{eV}$ where the gradient becomes weaker. The data are overlayed by calculated physical sputtering yields for normal incidence [8,9], where the energy of the impinging ions is taken as $E=2 k_{B} T_{i}+3 Z k_{B} T_{e}[10]$ assuming $T_{i}=T_{e}$. The curve with pure deuterium sputtering is clearly too low and tungsten is sputtered by impurities in accordance with measurements during the tungsten divertor experiment in 1996 [7]. The impurity and charge stage composition in the divertor is very difficult to assess, and Fig.4 just shows three model curves with an admixture of one extra impurity ion $\mathrm{C}^{4+}$ on a level of $1-4 \%$ as a placeholder for all low-Z impurity ions existing.

Spatial profiles of tungsten influx across the outer divertor were measured by simultaneous spectra recording on 12 lines-of-sight with time resolution of $3 \mathrm{~ms}$. The profiles cover the complete outer divertor region from the strike point area to the upper end of the baffle plates with a total distance along the poloidal surface contour of $0.48 \mathrm{~m}$. Integration of the influx density over the surface contour along the co-ordinate $S$ yields the total erosion rate at the outboard divertor: 
$\Phi_{\text {div }}=2 \pi \int \Gamma(S) R(S) d S$. The time resolution is too slow to reveal details of the ELM event. However, it is fast enough to determine the erosion profile in-between ELMs and to separate it from the erosion due to ELMs. Fig.5 shows temporally averaged influx profiles versus the distance of the observed spot to the position of the strike point along the divertor contour. The last channel at $S-S_{\text {sep }} \approx 0.51 \mathrm{~m}$ has almost the same signal as the channel at $0.33 \mathrm{~m}$ and is not included in the plots. The profiles of the upper box are from an improved H-mode discharge \#22920 with $I_{P}=1 \mathrm{MA}, q_{95}=4.8$, triangularity $\delta=0.3$, total heating power $P_{\text {heat }}=13.7 \mathrm{MW}$, low gas puff level of $\Phi_{D, p u f f}=4 \times 10^{21} \mathrm{~s}^{-1}$, and consequently low level of neutral flux density in the divertor of $\Gamma_{D}=5.0 \times 10^{22} \mathrm{~m}^{-2} \mathrm{~s}^{-1}$. The time averaged profile has the same shape as the profile between ELMs with a peak near the separatrix, where the temperature in-between ELMs has a value of about $20 \mathrm{eV}$. In the far SOL, the W influx slowly decays with distance away from the separatrix, however, the interpretation is difficult, since the flux surfaces are almost tangent to the poloidal divertor contour. The erosion in-between ELMs is as important as the erosion by ELMs. The ELMs have a frequency of $100 \mathrm{~Hz}$ and each ELM event produces $N_{E L M}=8.5 \times 10^{17}$ $\mathrm{W}$ atoms, representing $50 \%$ of the total outer divertor source rate $\Phi_{d i v}=1.7 \times 10^{20} \mathrm{~s}^{-1}$. The energy loss of the plasma is $\Delta W_{E L M}=41 \mathrm{~kJ}$ per ELM and thermography measures an energy deposition in the outboard divertor of $E_{d e p, E L M}=5.5 \mathrm{~kJ}$ per ELM.

The lower box of Fig.5 displays profiles for the improved H-mode discharge \#23060, which had similar parameters besides higher values for $\delta=0.33, \Phi_{D, p u f f}=1 \times 10^{22} \mathrm{~s}^{-1}$, and neutral divertor flux $\Gamma_{D}=9.3 \times 10^{22} \mathrm{~m}^{-2} \mathrm{~s}^{-1}$. The profile between ELMs has a different shape from the mean erosion profile and does not show the peak near the separatrix. Langmuir probes deliver in this case a lower temperature in-between ELMs of about $6 \mathrm{eV}$ near the separatrix. The total outer divertor source rate is much lower with a value of $\Phi_{d i v}=6.4 \times 10^{19} \mathrm{~s}^{-1}$ and $80 \%$ of it is due to ELMs. The ELMs have a frequency of $61 \mathrm{~Hz}$ and each ELM event produces $N_{E L M}=8.4 \times 10^{17}$ $\mathrm{W}$ atoms. In terms of energy, the ELMs are larger with $\Delta W_{E L M}=86 \mathrm{~kJ}$ and $E_{\text {dep,ELM }}=9.8 \mathrm{~kJ}$ per ELM.

It is not attempted to give a model for the ELM induced W erosion in the divertor, which would need to include the time development of $T_{i}, T_{e}$, the fluxes of deuterium and impurity ions and the charge state distributions of the impurities, however, an estimate for pure deuterium erosion shall be given and compared with the data. Assuming that $T_{e}$ is at pre-ELM values during the 
time when the ion flux arrives, pure deuterium sputtering does not seem to be sufficient to explain the number of sputtered $\mathrm{W}$ atoms during the ELM, since it would require that all D ions have a temperature of $T_{i} \approx 500 \mathrm{eV}$, i.e. to have just the temperature, where the number of sputtered $\mathrm{W}$ atoms per deposited energy has its maximum value of $3.1 \times 10^{16} \mathrm{~kJ}^{-1}$. Even then, the deposited ELM energy would have to be $\approx 26 \mathrm{~kJ}$, which is a factor of $2.7-4.8$ above the measured energy deposition. Even though this mismatch between model and measurement is not as strong as for the sputtering in-between ELMs, it appears that additional sputtering by impurities is also important for the erosion during ELMs.

\section{Main chamber tungsten sources and fueling}

The most important tungsten sources in the main chamber originate from the inner heat shield (HS) at the high field side, and the limiters at the low field side. The lines-of-sight used to measure the $\mathrm{W}$ influx from these components is shown in Fig.1 (see also Fig.1 in Ref.[11] for the limiter observation). The HS covers the inner column and has no openings that would cause leading edges and toroidally-nonuniform sources. The heat shield is monitored with a poloidal array of 9 lines-of-sight (time resolution $3 \mathrm{~ms}$ ). On the low-field side, the four ICRH antennas are surrounded by limiters forming a window frame. There are 17 lines-of-sight spread over three limiter sides and one top section of those window frames (exposure time of $3.4 \mathrm{~ms}$ ). All observed spots have a diameter of $\approx 3 \mathrm{~cm}$. There are also 4 protection limiters by the neutral beam ports that are not monitored for $\mathrm{W}$ influx.

At the central column, the poloidal erosion profile is rather flat for equilibria, where the poloidal shape of the flux surfaces matches the surface contour of the heat shield. However, for many plasmas, the SOL flux surfaces have a stronger curvature than the HS surface and touch the HS only near the equatorial plane. In such cases, the erosion happens mainly above the equatorial plane, while the signals from the lower part of the HS are very low [1]. The upper half of the HS is then shielding the lower half from the particle and power flux crossing the separatrix at the low-field side. We designate flux surfaces by $\Delta R$, the radial distance of the flux surface to the separatrix on the low-field side at the height of the magnetic axis. In that coordinate, the $\mathrm{W}$ influx decays below the equator with radial e-folding length of $\approx 0.25 \mathrm{~cm}$. The 


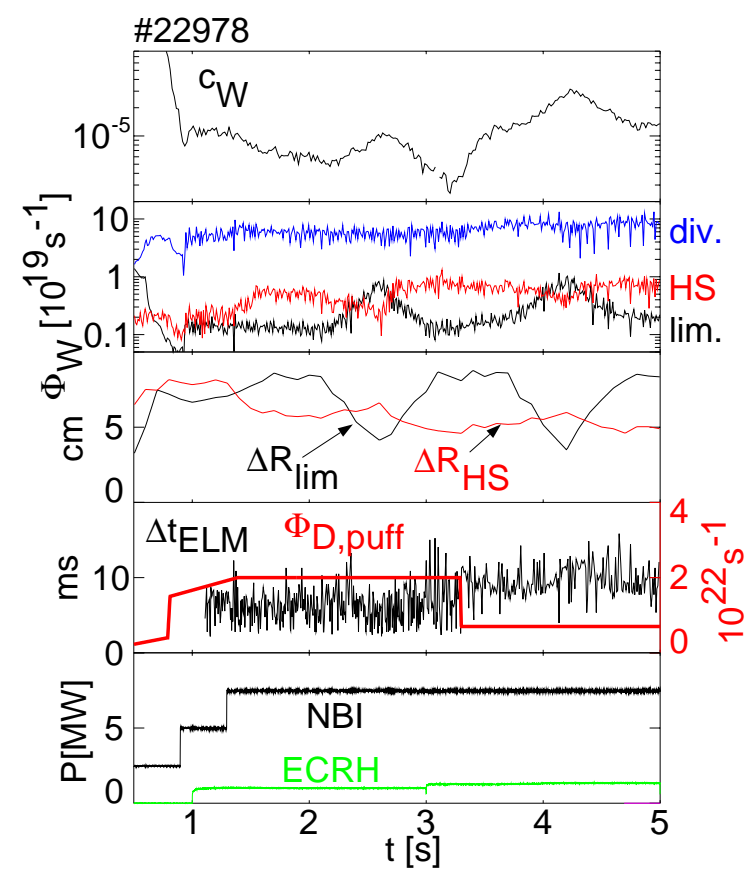

Fig. 6. Time traces for discharge \#22978 with variations of the outer separatrix radius at two levels of gas puffing.

total source rate $\Phi_{H S}$ is calculated by interpolating between the measured spots and integrating over the whole heat shield assuming toroidal symmetry. Typical source rates are $\Phi_{H S}=(0.5-$ $1.5) \times 10^{19} \mathrm{~s}^{-1}$. Again, a large fraction of this source, $(60 \pm 10) \%$, is caused by ELMs with a tendency to lower ELM contributions, when the separatrix is closer to the heat shield.

The poloidal influx profile for the vertical section of an outboard limiter is peaked where the surface is closest to the separatrix. The radial decay lengths are about $1 \mathrm{~cm}$, which is much larger than for the inner HS below the midplane. For the total source determination, the poloidal influx density profile from one limiter, which is observed with 9 lines-of-sight, is integrated using $5.5 \mathrm{~cm}$ for the toroidal width of the emission cloud of tungsten [12]. The signals from different limiters at same poloidal position do not differ very much and the integrals from those limiters, which are not as completely covered by measurement as the first limiter can be calculated by assuming the same poloidal and toroidal shapes. Information from 3 observed spots on the upper toroidal limiter of the window frame are used to add this part to the total integral. The lower window frame is estimated by scaling with the ratio of values from the lowest spot on the limiter to the value from the upper window frame (almost all equilibria have a lot higher distance to the lower part of the limiter and the influx at the lower end is small). The total influx from all limiting structures on the low-field side is estimated by multiplying the integral from 
the two antennas with a factor of 3 to account for the other limiters and window frames. Typical values without ICRF heating are $\Phi_{l i m}=(2-5) \times 10^{18} \mathrm{~s}^{-1}$. $(70 \pm 10) \%$ of the total limiter source is due to ELMs being in agreement with previous local measurements [13]. ICRF heating produce a strong local increase of the influx at the active antennas $[12,13]$. New results an this effect are given in Ref.[11].

The heat shield source is the larger source in the majority of the discharges. This is because it is usually on a flux surface with lower $\Delta R$ than the low-field side limiters, i.e $\Delta R_{H S}<\Delta R_{\text {lim }}$. Correspondingly, when the plasma column is shifted to the low-field side, such that the antenna frames become the first limiting structure, the behaviour is reversed. This is exemplified in Fig.6, where all tungsten source rates from divertor, heat shield and limiters are shown for a discharge with two sweeps of the outer separatrix radius. The source rates are not shown with full time resolution in order to get rid of the large ELM fluctuations. The effect of the radial plasma movement on $\Delta R_{H S}$ and $\Delta R_{\text {lim }}$ is shown in the third panel. When the time traces of the $\Delta R_{H S}$ and $\Delta R_{\text {lim }}$ cross, the corresponding source rates cross as well. The divertor source is about an order of magnitude larger, and it increases from $\Phi_{d i v}=5.8 \times 10^{19} \mathrm{~s}^{-1}$ to $\Phi_{d i v}=8.0 \times 10^{19} \mathrm{~s}^{-1}$ when reducing the puff level at $t=3.3 \mathrm{~s}$. The puff level, given in the 4 th panel of Fig.6, is the main actuator used at ASDEX Upgrade to change the frequency of type-I ELMs at a given heating power. A reduction of the gas puff leads to a hotter divertor and a longer ELM period. Here, the puff level was changed from $\Phi_{D, p u f f}=2 \times 10^{22} \mathrm{~s}^{-1}$ to $7 \times 10^{21} \mathrm{~s}^{-1}$ and the ELM period, which is shown in the forth panel, increased from $\Delta t_{E L M}=6.0 \mathrm{~ms}$ to $\Delta t_{E L M}=9.4 \mathrm{~ms}$. The main chamber sources also show a slight increase, however, this might be caused by the changes of $\Delta R_{\text {lim }}$ and $\Delta R_{H S}$. The strongest influence is seen on tungsten concentration $c_{W}$, which is determined from emission lines radiating at $T_{e}=1-1.5 \mathrm{keV}$, i.e. at about $r / a=3 / 4$ [14]. The $\mathrm{W}$ concentration rises by about a factor of 3 , when reducing the puff level. This is much stronger than the increase of all the sources and therefore, must be attributed to a transport effect. Here, the transport in the edge transport barrier, which is known to be characterised by an inward pinch for the impurities between the ELMs, is the decisive element $[14,15]$. Besides the change of $c_{W}$ with the ELM period, the concentration is varying in phase with the modulation of the source rate $\Phi_{l i m}$ from the low-field side limiters, even though $\Phi_{\text {lim }}$ has the lowest values of all the sources. Thus, the tungsten penetration from the low-field side plasma facing components is much more efficient than from the heat shield. We speculate that this is due to the shorter geometrical distance from 
the source location to the separatrix at the low-field side. Here, the distance is about $\Delta R_{\text {lim }}$ and on the high-field side, it is roughly $2 \times \Delta R_{H S}$ due to flux expansion. A lower level of the perpendicular transport, a higher prompt redeposition, and larger parallel flows at the highfield side might add to the asymmetry. In Alcator C-Mod, Mo [16] and low-Z impurities (N,C) $[17,18]$ also showed a much higher penetration at the low-field. Penetration of $\mathrm{W}$ injected by laser-blow off did not show much variation between low-field and high-field side in ASDEX Upgrade [19].

\section{Control of tungsten confinement in improved H-mode discharges}

Control of the tungsten confinement is essential for the achievement of improved H-mode discharges. The control has to work against the $\mathrm{W}$ accumulation in the centre of the plasma and to prevent the impurity transport from being predominantly neo-classical [20]. Presently, the only actuator is central ECRH, since ICRH produces a large extra tungsten source at the window frames around the antenna $[13,11]$. An ECRH power of (0.7-1.3) MW is usually used for this purpose. In H-mode discharges, the ELM frequency has to be kept at a sufficiently high level to control the tungsten penetration through the edge transport barrier.

Fig.7 concentrates on the second issue. It shows a series of improved H-mode discharges (\#22895, \#22898, \#22900, \#22901) at $I_{p}=1 \mathrm{MA}$, which have $1.3 \mathrm{MW}$ of central ECRH and $P_{N B I}=5 \mathrm{MW}$ up to $2.8 \mathrm{~s}$, when another NBI source with $2.5 \mathrm{MW}$ is switched on. The gas puff starts at a high value of $\Phi_{D, p u f f}=1.5 \times 10^{22} \mathrm{~s}^{-1}$ and is reduced at $2.5 \mathrm{~s}$ to a level between (1$10) \times 10^{21} \mathrm{~s}^{-1}$ as indicated in the figure. The ELM period, which is shown in the forth panel, starts at a low level. After the decrease of the gas puff, it increases with a stronger slope for stronger reductions of the gas puff. With the addition of the extra heating, it again decreases. This is a signature for type-I ELMs. The tungsten concentration at $r / a \approx 3 / 4$, which is depicted in the third panel, is also rising and shows a similar signature as the ELM period (the tungsten density shows the same time evolution, since the electron density is almost constant with slight variations between 8 and $8.5 \times 10^{19} \mathrm{~m}^{-3}$ ). In addition, the tungsten concentration has a modulation, which is introduced by two radial plasma sweeps, where the outer radius of the separatrix is shifted by $2.5 \mathrm{~cm}$ towards the low-field side limiters. This causes a rise of the tung- 


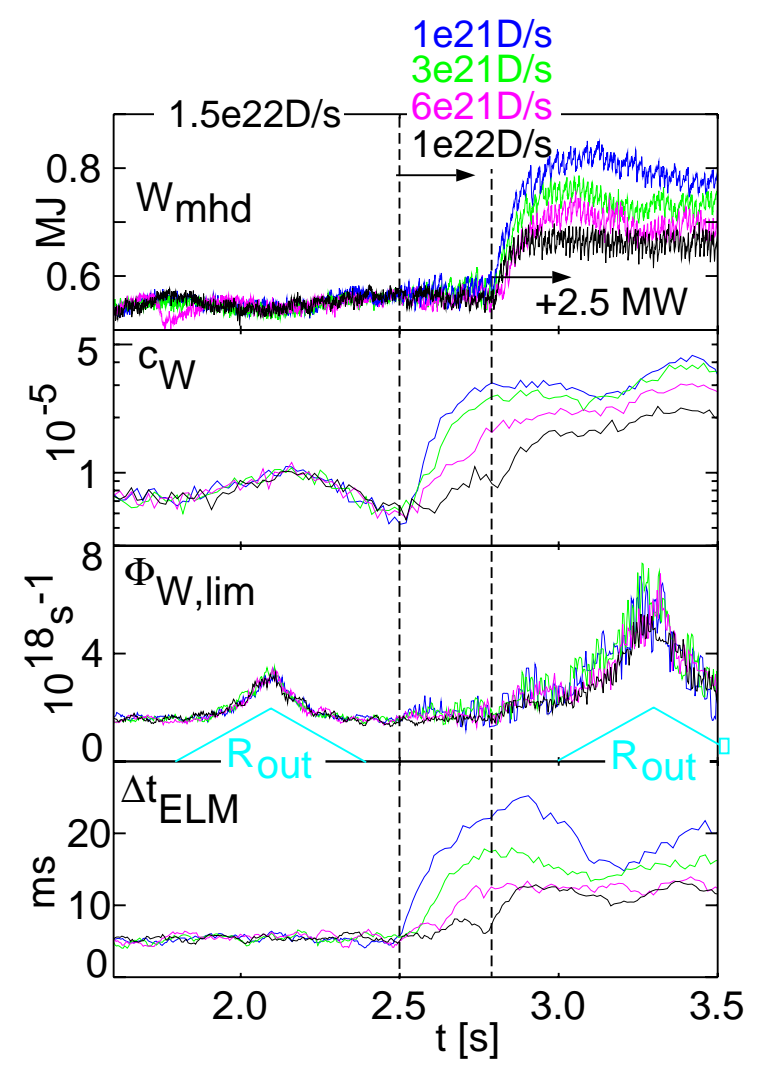

Fig. 7. Time traces for a series of H-mode discharges with a step in the gas puff level from a high initial value to four different reduced gas puffs at $2.5 \mathrm{~s}$. For the lowest puff levels, the longest ELM periods and the highest tungsten concentrations $c_{W}$ are observed.

sten erosion source $\Phi_{\text {lim }}$ at the limiters, which is a shown in the third panel (again without the full time resolution). The source rate is not influenced by the step-down of the gas puff, and is just increasing with the increase of the heating power. The heat shield source is on average 6 times higher than $\Phi_{l i m}$ with a decrease during the radial shift, and the divertor source is on average $26 \times \Phi_{l i m}$ with no change during the R-scan. Thus, like in the example from the previous section, the tungsten fueling seems to be dominated by the limiter source. The stored energy (top panel) reaches the highest values with the lowest gas puff. However, the discharge with the highest confinement has too high W concentration and evolves towards a strong central tungsten accumulation, which starts at $3.4 \mathrm{~s}$ and finally leads to a loss of confinement when the central ECRH is switched off. ELM periods of about $12 \mathrm{~ms}$ proved to be sufficient for stable operation, and by addition of an extra $2.5 \mathrm{MW}$ later in the discharge with $\Phi_{D, p u f f}=3 \times 10^{21} \mathrm{~s}^{-1}(\# 22900) \mathrm{a}$ stored energy of $940 \mathrm{~kJ}$ could be achieved with a tungsten concentration of $c_{W}=1.8 \times 10^{-5}$ and no central tungsten accumulation. This represents an $\mathrm{H}$-factor of $\tau_{E} / \tau_{I T E R H 98 y 2}=1.2$. 


\section{Conclusion}

Plasma operation in the non-boronised all-W ASDEX Upgrade could be extended to discharges with higher plasma current and higher heating powers. In improved H-mode discharges, all tungsten sources in the main chamber and in the divertor were quantified from simultaneous spectroscopic observation of 38 different positions. The erosion is mainly caused by ELMs and only in plasmas with a hot divertor, the erosion in-between ELMs is of same magnitude. The main erosion source is the outer divertor, however, the main tungsten fueling of the plasma is due to the erosion at the low-field side limiters. The ELMs are essential in reducing the inward transport of tungsten in the H-mode edge transport barrier. In most plasmas, the ELM frequency is accelerated by applying a higher gas puff, which reduces energy and impurity confinement at the same time. A careful balance is needed to achieve good $\mathrm{H}$-factors at low W concentrations. In the future, ELM triggering by small pellets might be used to independently control ELM frequency and recycling level. A control of central impurity transport by central ECRH is mandatory to avoid central tungsten accumulation in improved H-mode discharges.

\section{References}

[1] R. Neu, M. Balden, V. Bobkov, R. Dux, et al., Plasma Phys. Controlled Fusion 49 (2007) B59.

[2] M. Mayer, V. Rohde, K. Sugiyama, et al., J. Nucl. Mater. 390-391 (2009) 538.

[3] K. Schmid, T. Schwarz-Selinger, W. Jacob, et al., Nucl. Fusion 47 (2007) 984.

[4] M. Mayer, V. Rohde, G. Ramos, et al., Physica Scripta T128 (2007) 106.

[5] J. Roth, D. Naujoks, K. Krieger, et al., J. Nucl. Mater. 220-222 (1995) 231.

[6] K. Behringer, H. P. Summers, B. Denne, et al. Plasma Phys. Controlled Fusion 31 (1989) 2059.

[7] A. Thoma, K. Asmussen, R. Dux, et al., Plasma Phys. Controlled Fusion 39 (1997) 1487.

[8] W. Eckstein, C. García-Rosales, J. Roth, and W. Ottenberger, Technical Report IPP 9/82, MaxPlanck Institut für Plasmaphysik, Garching, Germany, 1993.

[9] W. Eckstein, Technical Report IPP 9/132, Max-Planck Institut für Plasmaphysik, Garching, Germany, 2002. 
[10] P. Stangeby, The Plasma Boundary of Magnetic Fusion Devices, Institute of Physics Publishing, Bristol and Philadelphia, 2000.

[11] V. Bobkov, F. Braun, R. Dux, et al., J. Nucl. Mater. 390-391 (2009) 900.

[12] R. Dux, R. Neu, V. Bobkov, et al., in Proc. of the 21st IAEA Conference Fusion Energy (CD-Rom), Chengdu, China, October 2006, volume IAEA-CN-149, pages EX/3-3Ra, Vienna, 2007, IAEA.

[13] R. Dux, V. Bobkov, N. Fedorczak, et al., J. Nucl. Mater. 363-365 (2007) 112.

[14] R. Neu, R. Dux, A. Kallenbach, T. Pütterich, M. Balden, et al., Nucl. Fusion 45 (2005) 209.

[15] R. Dux, Fusion Science and Technology 44 (2003) 708.

[16] B. Lipschultz, D. Pappas, B. LaBombard, et al., J. Nucl. Mater. 290-293 (2001) 286.

[17] G. McCracken, R. Granetz, B. Lipschultz, et al., J. Nucl. Mater. 241-243 (1997) 777 .

[18] G. McCracken, B. Lipschultz, B. LaBombard, et al., Phys. Plasmas 5 (1997) 1681 .

[19] R. Neu, V. Rohde, A. Geier, K. Krieger, H. Maier, et al., J. Nucl. Mater. 290-293 (2001) 206.

[20] R. Dux, R. Neu, C. F. Maggi, A. G. Petters, R. Pütterich, et al., in Proc. of the 20th IAEA Conference Fusion Energy (CD-Rom), Vilamoura, Portugal, November 2004, volume IAEA-CSP-25/CD, pages IAEA-CN-116/EX/P6-14, Vienna, 2005, IAEA. 